REVESCO. Revista de Estudios Cooperativos

ISSN: $1885-8031$

\title{
La tecnología Blockchain en la construcción de espacios económicos de impacto social positivo
}

\author{
Salvador Pérez Sempere ${ }^{1}$ y Antonia Sajardo Moreno ${ }^{2}(0)$
}

Recibido: 7 de julio de 2020 / Aceptado: 3 de diciembre de 2020 / Publicado: 18 de marzo de 2021

Resumen. Este trabajo realiza un estudio del universo de la tecnología blockchain y su aplicación en el mundo del siglo XXI a través del análisis de diferentes espacios virtuales construidos con ella. El objetivo último de este análisis es valorar y comprender las posibilidades que esta tecnología aporta en la construcción de procesos con un alto impacto social y/o ambiental. A tal efecto, se lleva a cabo una investigación exploratoria a través del estudio de casos significativos o pioneros desde una perspectiva socio-tecnológica. Los resultados concluyen que, si bien en sus orígenes la tecnología blockchain se estructuró con una marcada perspectiva financiera, el transcurso del tiempo ha demostrado su importancia real, su aportación y sus capacidades de descentralización, transparencia e inmutabilidad en espacios sociales con impacto social positivo.

La construcción, en base a las mencionadas propiedades, de una tecnología que cuenta con la confianza como un valor inherente y clave con el que se evita la necesidad de agentes intermedios, abre la puerta a su aplicación en procesos de transformación social. Ello conforma una visión alternativa de la tecnología blockchain que es aún minoritaria y que ha implicado ciertas limitaciones a la hora de establecer vínculos y obtener información sobre aplicaciones que sustenten tal visión. No obstante, y por esta misma razón, el grado de originalidad de este trabajo es elevado, lo que posibilita en última instancia sentar las bases de una perspectiva diferente a la hora de observar el uso de la tecnología blockchain.

Palabras clave: Blockchain; Innovación social; Impacto social; Transformación social.

Claves Econlit: O33; P46; P49; I31.

\section{[en] Blockchain technology in the construction of economic spaces of positive social impact}

\begin{abstract}
This work makes a study of the universe of blockchain technology and its application in the world of the 21 st century through the analysis of different virtual spaces built on it. The ultimate objective of this analysis is to assess and understand the possibilities that it provides in the construction of processes with a high social and / or environmental impact. To this end, an exploratory investigation is carried out through the study of significant or pioneering cases from a socio-technological perspective. The results conclude that, although blockchain technology was originally structured with a strong financial perspective, the passage of time has demonstrated its real importance, its contribution and its capacities for decentralization, transparency and immutability in social spaces with a positive social impact.

The construction, based on the aforementioned properties, of a technology that has confidence as an inherent and key value that avoids the need for intermediate agents, opens the door to its application in processes of social transformation. This forms an alternative vision of blockchain technology that is still a minority and that has implied certain limitations when establishing links and obtaining information about applications that support this vision. However, and for this same reason, the degree of originality of this work is high, which ultimately embraces laying the foundations for a different perspective when looking at the use of blockchain technology.
\end{abstract}

Keywords: Blockchain; Social innovation; Social impact; Social transformation.

Sumario. 1. Introducción. 2. La tecnología blockchain: estructuración y funcionamiento. 3. Metodología de investigación. 4. Análisis empírico: estudio de casos. 5. Conclusiones. 6. Nota de los autores. 7. Referencias bibliográficas.

Cómo citar. Pérez Sempere, S.; Sajardo Moreno, A. (2021) La tecnología Blockchain en la construcción de espacios económicos de impacto social positivo. REVESCO. Revista de Estudios Cooperativos, vol. 138, e73867. https://dx.doi.org/10.5209/reve.73867.

\footnotetext{
Universidad de Valencia, España.

Dirección de correo electrónico: voroperez@gmail.com.

2 Universidad de Valencia, España.
}

Dirección de correo electrónico: antonia.sajardo@uv.es. 


\section{Introducción}

La realidad del siglo XXI evidencia una larga lista de problemáticas y situaciones sociales y económicas, algunas de reciente aparición, que reclaman atención. La construcción de soluciones de cariz sistémico que posibiliten respuestas adecuadas representa un verdadero reto para nuestra sociedad, en algunos casos aún no suficientemente preparada. El envejecimiento de la sociedad, la despoblación del mundo rural y su consecuente concentración en entornos urbanos, la exclusión financiera también en los citados entornos, el cambio climático, las migraciones y las realidades que surgen con ellas, o el incremento estructural del desempleo, son solo una muestra de evidencias de las que progresivamente las sociedades van tomando conciencia y demandando soluciones a los gobiernos.

A nivel del Estado español, se observa como la estructura de edades de la sociedad ha ido variando a lo largo del tiempo encontrándose en un punto de estrechamiento en la base de la pirámide poblacional debido por una parte al descenso de la natalidad y por otra al incremento de la esperanza de vida (Pérez Díaz, 2010). La búsqueda de las causas de este proceso quedan más allá del interés de este artículo, pero sí resulta de interés observar cuáles son las principales consecuencias del mismo, a saber, la feminización de la sociedad, el sobre-envejecimiento y el aumento de la dependencia. Todos estos hechos generan la aparición de nuevas e importantes problemáticas como son el mantenimiento de un sistema de pensiones para la vejez, el incremento de necesidades en atención sanitaria, la prestación de cuidados por parte de los familiares, sin olvidar sus efectos sobre la competitividad del mercado laboral.

Otro de los fenómenos demográficos que, si bien se ha producido a lo largo de toda la historia, toma una relevancia especial en el contexto actual por su impacto en la composición del perfil demográfico de los Estados, es el de los flujos migratorios. Las personas migrantes constituyen un subconjunto poblacional que va a requerir de unos servicios específicos que le permitan alcanzar el objetivo por el cual han abandonado sus países de origen. Los motivos que producen este fenómeno migrante son de índole muy variada (Arango, 2003), no obstante, cuentan con un sustrato común consistente en la búsqueda de una mejora en la situación laboral o económica de la persona migrante, que indudablemente le aboca a una nueva situación de vida. En esta nueva situación uno de los aspectos más relevantes del colectivo migrante lo conforma la gestión de las llamadas remesas, concretadas en el envío de recursos económicos a la parte de la familia que queda en el país de origen, y con la que se comparte un porcentaje de los recursos económicos obtenidos en el nuevo destino (Grande, 2018). Al tratarse de flujos esencialmente privados y en muchas ocasiones informales, resulta complicado conocer con exactitud el volumen de los mismos, bien sean emitidos o bien sean recibidos por un Estado (Ibid.) No obstante existen estimaciones que sitúan el volumen de las remesas emitidas desde el Estado español en torno a los 17.874 millones de dólares ${ }^{3}$. Si bien estas transferencias monetarias tienen un impacto positivo en la lucha contra la pobreza en los países de origen (Tsvetanova, 2017), conllevan un problema fundamental que es el actual elevado coste de emisión de las mismas, coste que puede llegar a alcanzar entre un 10 y un $15 \%$ de su valor total (More, 2005).

En este mismo ámbito, el financiero, y en relación directa y transversal con las migraciones o la despoblación en el mundo rural, se presenta un problema adicional. Este no es otro que el de la exclusión financiera acaecida en el España principalmente por el proceso de fusión, para su posterior bancarización, de una gran parte de las Cajas de Ahorro y el redimensionamiento del sector que derivado. Ello ha implicado un proceso de cierre de numerosas sucursales bancarias consideradas como de menor rentabilidad económica, aunque situadas principalmente en municipios pequeños, a los que la banca tradicional no había llegado a tener presencia. El impacto directo sobre el bienestar de la sociedad ha sido más que evidente (Fernández et al., 2013). Así, el difícil acceso a los servicios financieros en las zonas rurales o en espacios urbanos desfavorecidos viene produciendo efectos económicos muy negativos, afectando directamente a grupos minoritarios que viven en estas áreas y que sufren situaciones de exclusión social (Zubaldia et al., 2008).

Simultáneamente, el contexto industrial actual se halla inmerso en la ya identificada Cuarta Revolución Industrial, surgida al calor de la introducción y la utilización de sistemas ciber físicos (Del Val Román, 2016). En su seno el número de trabajos susceptibles de ser automatizados se incrementa profusamente en toda la industria, especialmente en la manufacturera, disminuyendo consecuentemente el número de trabajadores necesarios para mantener su funcionamiento (Stock y Seliger, 2016). La desaparición de los trabajos de baja cualificación y reducida retribución, podría generar un desempleo estructural y al mismo tiempo un incremento de la desigualdad social (Zhenqian, 2018).

En este sentido Frey y Osborne (2017) realizan un riguroso análisis de las profesiones susceptibles de desaparecer por la computarización de la economía concluyendo que, en el mercado laboral de Estados Unidos existe el riesgo de desaparición del $47 \%$ de los empleos en los próximos 15 o 20 años. De igual manera el informe final del Foro Económico Mundial de Davos de $2016^{4}$ prevé la desaparición de 7,1 millones de empleos en los 15 países más industrializados del mundo, y la creación de 2,1 millones de

Volumen de las remesas emitidas desde el Estado español https://datosmacro.expansion.com/demografia/migracion/remesas/espana. Informe final del Foro Económico Mundial de Davos de 2016 https://www.weforum.org/agenda/2016/01/a-recap-of-davos-2016. 
nuevos puestos de trabajo para 2020, la mayoría relacionados con las nuevas capacidades y habilidades digitales (Kahale, 2017). La conclusión de todo ellos es clara: el modelo económico actual va a ser incapaz de ofrecer suficientes puestos de trabajo que permitan a amplias capas de la población obtener los recursos suficientes para cubrir sus necesidades básicas de subsistencia (Espinosa y Poza, 2018).

Y frente a ello el papel de las entidades de acción social, las cuales vienen atravesando una situación de escasez de recursos a raíz de las políticas aplicadas para gestionar la salida de la profunda crisis económica vivida a escala mundial a principios siglo (Esade et al, 2013). Así, la ya mencionada desaparición de las cajas de ahorro, y con ellas de sus donaciones a proyectos de acción social, ha venido provocado el cese de actividad de numerosas entidades (Ruiz, 2015), poniendo en serios problemas de viabilidad a un gran número de entidades y proyectos (Pérez, 2017). En todo ello ha influido igualmente una crisis de confianza general (Roth, 2009) que afecta entre otras instituciones a las Organizaciones No Gubernamentales (ONG) (Edelman, 2019) y que produce un impacto negativo en el volumen de donaciones procedentes de la ciudadanía (Pérez, 2017).

Una de las vías de solución que la propia tecnología ha ofrecido en el ámbito de la financiación de entidades sociales, y por ende de proyectos o emprendimientos vinculados, ha sido la utilización de la herramienta del crowdfunding, que permite el acopio de recursos financieros a partir de pequeñas donaciones de un gran número de donantes. No obstante, esta modalidad de financiación requiere del uso de plataformas ofertantes de servicios de crowdfunding que precisan de un porcentaje de donaciones para su mantenimiento, así como de servicios de pago on line ofertados por entidades financieras (Sajardo y Pérez, 2016), dándose la paradoja de que, en ocasiones, la recaudación de recursos por parte de las entidades sociales genera un flujo de comisiones cuyos beneficiarios pueden ser parte causante de la problemática objeto de atención (Ibid).

Todo lo anterior viene abocando a una crisis de confianza que pone en cuestión los sistemas sociales, políticos y económicos actuales que asisten al surgimiento y expansión de grupos sociales, de distinta índole, que ve con recelo el funcionamiento y los resultados derivados de la economía de mercado. Para esta parte de la ciudadanía la globalización es observada como una amenaza y un desafío que exige a los gobiernos un mayor control y una regulación más estricta en todos los sectores industriales (Roth, 2009).

A raíz de la desconfianza generada en el sistema financiero surgida tras la caída de Lehman Brothers en el año 2008 (García, 2015), el gurú tecnológico, Satoshi Nakamoto presenta una herramienta que va a permitir la realización de pagos online entre pares que serán enviados desde una parte a la otra sin la intervención de ninguna institución financiera (Nakamoto, 2008:1) Este sistema no es otro que el Bitcoin, la primera criptomoneda que, utilizando diferentes técnicas ya existentes como es el software que da soporte a aplicaciones P2P y la criptografía entre otras, es capaz de crear un entorno de confianza en el que se producen transferencias de valor entre pares sin la necesidad de la participación de una tercera parte, que por lo general suele ser una entidad financiera (Ibid). La tecnología sobre la que se ha construido el Bitcoin, y que está siendo utilizada para desarrollar nuevas aplicaciones en campos muy diversos de la vida, es conocida como tecnología de la cadena de bloques, más comúnmente denominada como blockchain.

El Bitcoin ha constituido el primer activo digital que no tiene un valor intrínseco, ni cuenta con ningún tipo de respaldo por un tercero, y que al mismo tiempo no precisa de una entidad centralizada emisora del mismo (Buterin, 2013). Asimismo, ha sido la primera propuesta surgida al abrigo de la tecnología blockchain.

Posteriormente, vista la potencialidad de aplicaciones que ofrecía Bitcoin, y no únicamente como herramienta financiera, los desarrolladores informáticos se encontraron con un dilema debiendo decidir entre la construcción de estas aplicaciones sobre el propio Bitcoin o hacerlo desde un nuevo blockchain. Es por ello que a finales del año 2013, un desarrollador de software entusiasta de Bitcoin llamado Vitalik Buterin lanza un white paper en el que presenta la idea de Ethereum, un blockchain con un propósito general que añade la posibilidad de ejecución de fragmentos de código (Antonopoulos y Wood, 2018). Se construye así el llamado ordenador mundial, una máquina virtual que se ejecuta en múltiples ordenadores conectados a la blockchain de Ethereum y que le proporciona capacidad de ejecución de un código informático a modo de pequeños programas que se denominan smart contracts, contratos inteligentes. Este código recoge las condiciones que deben darse para la ejecución de una transferencia, bien sea económica o bien de datos.

La tecnología blockchain es catalogada por Iansiti y Lakhani (2017) como una tecnología fundamental que tiene potencial para crear nuevos fundamentos para nuestro sistema económico y social. Así dota de nuevos contenidos al concepto de la cripto economía. El conjunto de características sobre las que se basa esta tecnología hacen que, como subraya el Comité Económico y Social Europeo $(2019)^{5}$, la tecnología blockchain tenga un encaje adecuado en la Economía Social, afirmando que "algunas características de estas tecnologías hacen de la cadena de bloques una infraestructura digital que podrían utilizar con provecho las organizaciones de la Economía Social para mejorar el cumplimiento de sus fines, incrementando su capacidad para generar un impacto social positivo y promover la innovación social”. 
Sobre esta base, el objetivo de este trabajo consiste en analizar el modo en que la tecnología blockchain está siendo utilizada de facto para la creación de aplicaciones descentralizadas (Dapps) que dan soporte a proyectos con un impacto social y ambiental relevante, valorando sus potencialidades para las entidades de Economía Social, a través del estudio de casos. A tal efecto, y más allá de una presentación amplia de la tecnología blockchain en su concepción y funcionamiento, se hace necesaria una síntesis de sus principales características para facilitar la comprensión de aquello que esta tecnología aporta a cada una de los casos que van a ser analizados, y que conforman el núcleo del presente análisis. Un apartado dedicado a la metodología seguida precede a los resultados del trabajo, que concluye con un apartado donde se recogen sus principales aportaciones.

\section{La tecnología blockchain: estructuración y funcionamiento}

Como ya ha sido indicado, una de las principales consecuencias de la crisis económica internacional que siguió al hundimiento en septiembre de 2008 del banco Lehman Brothers (García, 2015), ha sido la aparición de una crisis de confianza por parte de la ciudadanía tanto en los bancos y en la estabilidad global del sistema financiero, como en las instituciones y sus valores subyacentes (Roth, 2009).

Paralelamente, y en el citado marco de débil confianza internacional, un grupo de desarrolladores informáticos agrupados bajo el pseudónimo de Satoshi Nakamoto presentaron mediante la publicación de un paper en la Cryptography Mailing List, ${ }^{6}$ un sistema electrónico de transferencias de valor entre pares, que no precisaba de agentes intermedios que lo dotasen de confianza, ya que ésta estaba implícita en el propio sistema (Nakamoto, 2008). Este sistema se hallaba basado en la asignación de valor a un activo digital, que posteriormente podía ser utilizado para la realización de transferencias de su valor implícito entre pares de un modo irreversible, siendo registrada en una base de datos descentralizada e inmutable, gracias a la utilización de diversas tecnologías informáticas de control. El Bitcoin, que es el nombre que recibió este activo digital, irrumpió en el escenario tecnológico-financiero como la primera criptomoneda sin valor intrínseco y sin ninguna institución que le ofreciera respaldo (Buterin, 2013).

Todo este sistema fue sustentado sobre la tecnología conocida como blockchain. Blockchain consiste en una base de datos descentralizada en la que se registran todas las transacciones de valores o de datos que se produzcan entre las partes participantes en el sistema. Cada transacción es verificada y registrada en esta base tras el consenso de la mayoría de los participantes en el sistema (Crosby et alt., 2016). Las transferencias son agrupadas en bloques para su verificación, bloques que son colocados en cadena. Es decir, cada bloque está enlazado tanto con el bloque precedente como con el posterior, de ahí la denominación de la tecnología como blockchain o cadena de bloques.

Un concepto de especial importancia en este entorno es el del token digital, que es concebido como la representación digital de cualquier valor, creada por una entidad concreta y que puede ser utilizado de formas diversas gracias a la gran cantidad de información que puede contener. Así puede ser usado para la representación de una criptomoneda, para otorgar un derecho o para representar un bien físico (Rohr y Wright, 2017).

Los pasos de funcionamiento de un blockchain que cuenta con "n" nodos, entendiendo por nodo cada uno de los ordenadores que forman parte de la red, pueden ser esquematizados de la siguiente forma:

1. Dos nodos de la red deciden realizar un intercambio de valor o de datos a través del sistema blockchain al que se encuentran conectados. Para su realización efectiva difunden los datos de la misma al resto de nodos que están en conexión.

2. Los nodos conectados reciben esta transacción y la incluyen, junto con otras que hayan sido recibidas y que estén pendientes de realizarse, en un bloque. Este bloque se transmite posteriormente al resto de la red.

3. Los nodos evalúan las transacciones incluidas en el bloque y una vez validados, los nodos conectados utilizan mecanismos de consenso en base a un conjunto de reglas acordadas. Cuando se alcanza el consenso, que precisa del acuerdo de un número de nodos superior al $51 \%$ de aquellos que están conectados, las transacciones incluidas en el bloque son verificadas.

4. Una vez validado el bloque se aplica sobre él una función criptográfica de una sola dirección que devuelve un valor (denominado hash) que va a poder utilizarse para detectar cualquier alteración posterior del contenido del bloque. Cada bloque contiene un enlace al hash del bloque anterior, creando así una cadena de bloques inalterable gracias a las propiedades de la función hash(x).

De este modo se obtiene un sistema que proporciona seguridad, anonimato e integridad en los datos que utiliza, y de un modo independiente, es decir, sin necesidad de terceros actores que controles y certifiquen 
los mismos. Se elimina así, de un modo paralelo, la concentración de poder de análisis y de decisión (YliHuumo et al., 2016).

La gestión de los activos digitales, que son piezas clave del funcionamiento de la cadena de bloques, se realiza mediante la utilización de billeteras digitales, referidas habitualmente como wallets, por parte de las personas usuarias. Estas billeteras son piezas de software que permiten a las personas usuarias de una cadena de bloques monitorizar transferencias, almacenar y gestionar los activos digitales relacionados con la misma. Pueden asimismo estar conectadas continuamente a internet (hot wallets) o permanecer offline, de un modo aislado a la cadena de bloques (cold wallet) almacenando los activos en papel, una pieza de hardware o una unidad USB de almacenamiento externo (Antonopoulos y Wood, 2018).

En 2013 el desarrollador informático Vitalik Buterin propuso a la comunidad de desarrollo de Bitcoin la introducción de modificaciones en el sistema de scripting de Bitcoin que dotase de capacidad de ejecución de programas informáticos complejos. Así aparece la plataforma Ethereum que, utilizando la tecnología blockchain, crea un espacio descentralizado de código abierto con capacidad de ejecución de fragmentos de código con los que se implementan contratos inteligentes. Estos contratos inteligentes consisten en fragmentos de código construidos siguiendo las condiciones marcadas en un contrato entre partes, y que se ejecuta automáticamente cuando estas condiciones son alcanzadas.

El blockchain de Ethereum admite dos tipos de transacciones, aquellas que transfieren valor a mediante el uso de una criptomoneda y adicionalmente otras que realizan transferencias de datos. Estas últimas son las utilizadas para la ejecución de contratos inteligentes, que es realizada en la máquina virtual que Ethereum (EVM) construye con los nodos conectados (Antonopoulos y Wood, 2018). EVM es una suerte de ordenador global que contiene millones de fragmentos de objetos ejecutables, cada uno de los cuales tiene su propio almacén permanente de datos (ibid.).

Un elemento adicional que contribuye al potencial de uso de la tecnología blockchain son las aplicaciones descentralizadas (DApps). Las DApps son la base de la Web3, un entorno de aplicaciones descentralizadas que lo dotan de resiliencia, transparencia y resistencia a la censura (Ibid.). Se trata de aplicaciones que se ejecutan en internet de un modo descentralizado, sin la presencia de un agente central (Middlemen) que supervise su funcionamiento o gestione la información relativa a los usuarios (Johnston et al., 2014).

Otro importante concepto, que surge con la aplicación de las herramientas que la tecnología blockchain proporciona son las Organizaciones Descentralizadas y Autónomas (DAO, por sus siglas en inglés). Una DAO es una organización que, bajo un conjunto de reglas predeterminadas, dirige un negocio o una actividad social (tanto on line como off line) de un modo completamente autónomo en un entorno de código abierto que es descentralizado (distribuido entre los ordenadores de los stakeholders), transparente, seguro y auditable. Se trata de un grupo de contratos inteligentes y/o agentes autónomos unidos entre sí y dotados de un capital inicial (Wüst y Gervais, 2018:6).

Sobre esta base, numerosas criptomonedas se han construido principalmente con el objetivo de obtener los recursos financieros necesarios para la puesta en marcha de proyectos empresariales, principalmente basados en el desarrollo de aplicaciones en el ámbito de internet, y ser utilizadas como el medio de pago utilizado en los mismos. El blockchain introduce así herramientas y procedimientos que cambian el modo en que las personas organizan sus actividades económicas y entornos sociales, permitiendo el desarrollo de nuevos esquemas de gobernanza basados en una toma de decisiones más democrática y participativa.

Cada una de estas propuestas tiene como base de partida la redacción de un documento en el que se incluyen las características técnicas y de modelo de negocio que se propone en torno a cada proyecto. Para ello se utiliza la expresión white paper, que suele ser la base inicial de captación de los recursos que hagan posible el desarrollo de la propuesta, habitualmente a través de una Initial Coin Offerings (ICO). Se trata ésta de una emisión de criptomoneda que sale a la venta con el objetivo de captar financiación para la puesta en funcionamiento de proyectos basados en la tecnología blockchain. El concepto es similar a las Initial Public Offering (IPO) que lanzan las compañías en su salida a bolsa, pero adaptado a la realidad de la criptoeconomía.

La potencialidad de las múltiples aplicaciones que la tecnología blockchain tiene en ámbitos muy diversos hace que sea observada y comparada con la aparición de internet. Las predicciones sobre esta tecnología apuntan a que será un elemento clave en procesos de desplazamiento del equilibrio de poder desde un modelo centralizado, controlado por autoridades concretas, hacia una nueva situación donde el poder sea distribuido y utilizado en un sinfín de áreas como las comunicaciones, los negocios e incluso en los ámbitos políticos y legales (Wright y De Filipi, 2015).

Si bien esta tecnología se encuentra todavía en una fase muy incipiente, existen ya propuestas de construcción de soluciones a temas muy diversos en base a la utilización de las herramientas que pueden ser construidas mediante su uso. Son aplicaciones relativas tanto a la industria financiera, el mercado de valores o los seguros entre otras, como a entornos no financieros, como son las notarías públicas, la logística, la energía, la industria musical o el internet de las cosas (Crosby et al, 2016).

Uno de los campos más innovadores en los que esta tecnología puede ser aplicada es el referente a la Renta Básica Universal (RBU, en adelante). En este ámbito el blockchain constituye el andamiaje desde el 
cual se construyen ecosistemas económicos alternativos basados en la creación de comunidades que comparten una serie de normas y procedimientos que los configuran. Si bien desde la ortodoxia social y económica este tipo de propuestas se ven ralentizadas por un determinismo económico centrado en modelos meramente matemáticos y posturas ideológicas contrarias a modificar el orden social existente, surgen personas y entidades que, basándose en su percepción del valor final de la implementación de un sistema de RBU y su impacto en la vida, crean e impulsan proyectos de RBU al margen de la acción institucional.

\section{Metodología de investigación}

La reciente irrupción de la tecnología blockchain en el escenario tecnológico, ha abierto la puerta a la aparición de nuevas aplicaciones capaces de dotar de un entorno de confianza a procesos que hasta ahora venían dependiendo de actores terceros que avalaban y generaban, de una u otra forma, el citado espacio fiable de actuación. Pese a la rápida dinámica de desarrollo de esta tecnología, su aún corto recorrido impele a la necesidad de utilizar un enfoque exploratorio de carácter cualitativo y cuantitativo simultáneamente como aproximación metodológica de investigación.

Sobre esta base, este trabajo se ha centrado en el estudio de casos de diferentes propuestas de utilización de la tecnología blockchain, desde una perspectiva socio-tecnológica, a través de lo cual valorar las posibilidades que la utilización de esta tecnología abre al Sector de la Economía Social y Solidaria en la generación y desarrollo de proyectos con impacto social y ambiental positivos.

Yin (1989), Martínez Carazo (2006) y Villarreal \& Landeta (2010) dan fundamento a la utilización de la citada metodología en base a posibilitar el análisis del fenómeno objeto de estudio a través de fuentes de evidencia diversas, cuantitativas y/o cualitativas simultáneamente. Se posibilita así la construcción de una teoría mediante el análisis de una muestra teórica conformada por uno o más casos, en lugar de hacerlo desde la extracción de una muestra representativa, necesaria en un estudio cuantitativo (Martínez Carazo, 2006). En este sentido Yin (1989) considera el estudio de casos como una herramienta valiosa de investigación, al ser la vía utilizada para valorar y registrar conductas de los actores presentes en el fenómeno estudiado, en contraposición con métodos cuantitativos centrados en información verbal obtenida a través de encuestas por cuestionarios.

Así, el trabajo empírico de este estudio parte de un proceso de identificación, observación e interacción del conjunto de iniciativas que utilizan la tecnología blockchain en sectores de actuación social y medioambiental con potencial impacto positivo. Tras ello, se procede a una selección de un número reducido de iniciativas, o casos, para su posterior exploración y análisis con rigurosidad y profundidad. Los casos han sido seleccionados por su representatividad, tanto en términos de pionerismo, como en relación a su significación cuantitativa, o bien por su potencial cualitativo en el contexto de actuación en el que han surgido y se han desarrollado.

Las variables empleadas para la selección de los casos objeto de análisis se han basado en tres factores principales:

1. El ámbito de actuación desplegado para el abordaje de la problemática a la que van dirigidas, primando aquellas que no se desarrollan en un ámbito geográfico concreto, sino que despliegan su actuación de manera global. Si bien los casos estudiados no son los únicos existentes que cumplen este requisito, sus dinámicas ya han sido estudiadas en otros trabajos por las autoras del presente artículo, motivo por el cual el punto de partida de conocimiento es más elevado.

2. La existencia de espacios de actuación preeminente con presencia de desarrolladores de aplicaciones descentralizadas que utilizan la tecnología blockchain.

3. Su carácter innovador o pionero en la utilización de la tecnología blockchain en su ámbito de actividad y fuera de las aplicaciones financieras tradicionales. Ello se torna de suma importancia para valorar de qué modo aportan una visión que cuestiona el sistema socio-económico dominante en cuyo seno han aparecido, cumpliendo con ello con una de las funciones principales de la Economía Social, como es su vector político de pionerismo en la generación de iniciativas sociales inexistentes (Sajardo, 2012).

En términos procedimentales la labor de captación informativa se ha desarrollado en tres etapas. En una primera etapa se ha procedido a la revisión y análisis de los white paper que cada una de las iniciativas o casos seleccionados presenta en sus páginas web. Una segunda etapa se ha dedicado a la interacción con estas herramientas a través de su utilización como usuarias tecnológicas activas. Por último, se ha acometido una serie de contactos con los responsables de los casos objetivo, a través de conversaciones abiertas bien vía correo electrónico, twitter, mensajería instantánea y videoconferencias.

Los casos seleccionados para su estudio y análisis han sido los siguientes. En el campo de la energía se ha optado por la propuesta presentada por la plataforma Pylon Network por ser una herramienta dirigida a su utilización por parte de cooperativas de consumidores y usuarios que trabajan en la producción y 
comercialización de energías renovables. Al mismo tiempo actúa como elemento posibilitador del desarrollo de iniciativas de autoconsumo colectivo, una de las claves de la construcción de un nuevo modelo energético que se presenta como alternativa, en condiciones de mercado acordes con la competencia, y frente al oligopolio de grandes empresas energéticas que dominan el mercado en el Estado español en esta segunda década del siglo XXI.

En segundo término, se recoge la plataforma Arcadia, dirigida a la provisión de servicios financieros a personas refugiadas, que quedó en primera posición en el Startup Days Bern 2019 Pitching Battle. Este premio dió visibilidad a su propuesta y fue una de las 8 startup seleccionadas para participar en el_Fintech Solutions for Refugees Summit ${ }^{7}$ organizado por Village Capital ${ }^{8}$ y PayPal $^{9}$. Si bien existen en este ámbito otras propuestas que podían haber sido seleccionadas para su análisis, la plataforma Arcadia ha sido elegida por su accesibilidad y disposición a compartir información.

Adicionalmente, se ha seleccionado a Binance.charity que es una plataforma de crowdfunding propuesta por binance.com, uno de los principales exchanges de criptomonedas que propone y crea la Blockchain Charity Foundation con el objetivo de mejorar la transparencia en las donaciones filantrópicas, expandir la utilización de las criptomonedas y acelerar el desarrollo global sostenible. Su elección viene motivada por su carácter pionero en este espacio de actividad.

Por último, se han analizado la evolución y operativa de dos de las tres propuestas que desde el 2018 trabajan en el ámbito de la generación de la Renta Básica Universal (RBU), como son UBU y Mannabase. A través de su estudio se ha podido captar y analizar los cambios que se han producido en su misión desde una "perspectiva constructiva" propia del carácter multietápico y cíclico que se produce en los procesos de innovación y más específicamente en la innovación social. Procesos construidos sobre evidencias de prueba y error que introducen perspectivas diferentes para alcanzar el objetivo final que no varía.

En este contexto se ha recabado y analizado toda la información ofrecida por la iniciativa (también conocida como experimento) Gooddollar, que comienza su ejecución a finales de ese mismo año con el objetivo de evaluar la viabilidad de la utilización de la tecnología blockchain para reducir la desigualdad económica en la sociedad del siglo XXI. El método de recogida de información seguido se ha basado en la realización de test periódicos y cuestionarios dirigidos a personas que voluntariamente ponen sus dispositivos móviles a disposición del proyecto instalando una versión Beta (o versión de testado de funcionamiento del material que se va programado), así como su compromiso de implicación a interactuar periódicamente, asegurando así su tráfico y la verificación de su funcionamiento.

\section{Análisis empírico: estudio de casos}

\subsection{Democratización de la producción energética}

Los retos existentes en el sector energético a nivel global, y específicamente en el eléctrico, pasan por una evolución hacia un modelo de generación "de bajas emisiones, que asegure el suministro y la competitividad de la economía al mismo tiempo" (Costa, 2016:140). Al mismo tiempo se observa como la falta de comunicación entre los distintos actores que conforman el sector y el acceso desigual a los datos genera desconfianza entre ellos, y dificulta su cooperación en pro de la mejora del sector en su globalidad (Pylon Networkk, 2018). En un contexto de evolución de redes energéticas descentralizadas se hace necesario equilibrar la demanda de energía con la oferta de un modo inteligente, y para este logro la tecnología blockchain conforma una punta de lanza aportando la velocidad, la automatización y la descentralización necesarias (Schneiders y Shipworth, 2018).

En este ámbito, el energético, se ha seleccionado y analizado el caso de Pylon Network. Este proyecto se define en su white paper como "una base de datos energéticos neutral, que utiliza tecnología blockchain Open-Source, especialmente diseñada para satisfacer las necesidades del sector energético. Se trata de un código blockchain que es rápido, escalable y con requisitos mínimos de energía, diseñado para desempeñar el papel de la infraestructura de comunicación digital para una mayor participación de los activos distribuidos de los mercados energéticos, así como la prestación de servicios digitales de energía en el futuro de nuestros sistemas energéticos".

Pylon Network se presenta así como una plataforma descentralizada, en la que los diferentes stackeholders del sector energético (a saber, consumidores, comercializadoras, distribuidoras, productores energéticos) cuentan con la posibilidad de "volcar" la información sobre su propio historial de operaciones, e igualmente van a poder acceder a ella en base a su disposición en condiciones de código abierto, y con una mayor seguridad y transparencia. En todo momento el proyecto se concibe como una solución de mejora

Página web de Fintech Solutions for Refugees Summit: https://vilcap.com/current-programs/fintech-solutions-for-refugees-2019.

Página web de Village Capital: https://vilcap.com/.

Página web de PayPal: https://www.paypal.com/es/. 
orientada al cliente final, con el objetivo de habilitar un mercado energético más transparente abierto y competitivo para e 1 usuario final.

El objetivo último de este proyecto es posibilitar que las empresas oferentes de servicios energéticos realicen un análisis de los mismos y construir, en base a los resultados alcanzados, un conjunto de servicios que les permitan una utilización más eficiente de la energía. Con ello se persigue generar un uso energético eficiente, que va a determinar un ahorro en costes económicos por la energía consumida.

Para ello Pylon Network pone a disposición de las personas usuarias una app que les permite acceder a sus datos energéticos y compartirlos con empresas proveedoras de servicios energéticos. Esta herramienta forma parte de la amplia gama de instrumentos a través de los cuales se viene edificando un ecosistema alternativo formado por cooperativas de consumidores y usuarios con propuestas como somenergía o goiener, todas agrupadas bajo la cooperativa de segundo grado denominada Unión renovables que trabaja por un nuevo modelo energético en el Estado español.

El algoritmo de blockchain utilizado en Pylon Network, Pylon Coin CORE, ha sido diseñado específicamente para el sector energético, y pretende con ello cubrir las necesidades de escalabilidad, seguridad y privacidad, así como de sostenibilidad en base a unos costes energéticos mínimos.

Las características técnicas Pylon Coin Core se encuentran accesibles a través de la plataforma Github ${ }^{10}$, siendo relevante resaltar los aspectos críticos que se han tenido en cuenta en el diseño del mencionado algoritmo, como son la huella de carbono que conlleva su operativa y la escalabilidad del sistema. La coherencia con los citados aspectos críticos fundamenta una propuesta con visión cooperativa y no competitiva en el proceso de minería de los bloques, determinante para alcanzar un consumo energético mucho más bajo por transacción que los existentes en otros protocolos de consenso.

Si bien la herramienta es accesible a cualquier operador del sector energético, independientemente de la forma jurídica que este tenga, Pilon Network aspira a la democratización de la información, y a la consecuente construcción de un ecosistema cooperativo que permita una descentralización real del sector. De hecho, y en coherencia con ello, en marzo de 2018 se llevó a cabo una prueba piloto dentro de la cooperativa Goiener. Para ello se instalaron dispositivos de medición inteligentes que permitían adquirir los datos de consumo en tiempo real, realizar un trazado del origen de la energía que se consuma, y poder así pagar intercambios de energía entre usuarios que participen en esta red descentralizada.

Según Unión Renovables (unión de cooperativas de personas consumidoras y usuarias de energías renovables): "El movimiento cooperativo energético ha surgido en los últimos años en toda Europa como respuesta a la defensa del suministro energético como un bien básico libre de especulaciones, además del incentivo y promoción del uso de energías renovables".

\subsection{Crowdfunding}

A finales de los años noventa del siglo pasado, las herramientas de las tecnologías de la información y comunicación fueron introducidas en propuestas de financiación colectiva de variada índole. Esta modalidad de financiación, comúnmente conocida como crowdfunding, consolida pequeñas aportaciones, en relación con el monto global perseguido, procedentes de múltiples donantes para cubrir las necesidades económicofinancieras de determinados proyectos propuestos por persona o entidades concretas (Pérez, 2017).

Entre ellas, se han venido generando un sinfín de proyectos dirigidos a la acción social, que en ocasiones se enfrentan con una doble problemática, la técnica y la ideológica. En la parte técnica la utilización de las plataformas que proveen servicios de crowdfunding implica el pago a estas de un porcentaje de los fondos recaudados para su mantenimiento que suele oscilar entre el 4 y el $8 \%$ del monto total recaudado' y por otra la utilización de pasarelas de pago que introducen un coste adicional al montante donado por las personas participantes en el proyecto. Adicionalmente a esta realidad, y en estrecha relación con ella, se limita igualmente el monto mínimo de donación que de sentido al pago adicional de comisiones que cada aportación conlleva.

En la mayoría de los casos esta modalidad de financiación precisa de un factor de confianza en la entidad o en los gestores de la misma y a la cual se le aportan recursos, ya que este funcionamiento no hace viable la realización de un rastreo sobre el destino final de los fondos aplicados. Bien entendido de que siempre queda la posibilidad de obtener, dentro de una actitud voluntaria de buenas prácticas por parte del receptor de las donaciones, un informe final del proyecto que avale ex post que su uso ha sido aquel para el cual fueron aportados los recursos.

Desde la perspectiva ideológica, y teniendo en cuenta estos pagos colaterales que surgen por el mero hecho de participar en una campaña de crowdfunding tradicional, en ocasiones se da la paradoja de que las comisiones bancarias que se abonan van a parar a las arcas de entidades financieras que juegan un rol en este esquema de funcionamiento también determinante de la problemática que se pretende abordar. Así por

10 Github es una plataforma de desarrollo colaborativo de software. 
ejemplo, piénsese en proyectos que intentan atender a personas víctimas de los conflictos bélicos, y que con la creación y gestión de una campaña de crowdfunding a tal efecto, generan un flujo de comisiones hacia entidades financieras que, posiblemente, estén utilizando esos mismos fondos para la financiación de empresas productoras de las armas que son utilizadas en este mismo conflicto.

No obstante, la aparición de la tecnología blockchain y su utilización en esquemas de financiación colectiva de proyectos de acción social soluciona la mayor parte de estas problemáticas. El uso de criptomonedas puede minimizar el importe de los posibles pagos a terceros y añade un factor de confianza respecto del destino final de la donación, puesto que posibilita la trazabilidad de la misma (Acuña, 2018). Este factor de confianza es aportado por la propia naturaleza de la tecnología blockchain, que habilita un sistema de rastreo que permite conocer en qué gasto ha sido aplicado cada una de las unidades de valor que han sido donadas.

La posibilidad de dividir cada unidad de criptomoneda en valores que ocupan posiciones decimales muy alejadas de la unidad, como muestra puede observarse el bitcoin (BTC) y un satoshi o unidad mínima de medida de valor que equivale a $10^{-9} \mathrm{BTC}$, otorgándoles un valor ínfimo si son observadas de un modo aislado, abre la puerta a la aparición de nano donaciones. Sin embargo, si esta modalidad de donación se observa en un entorno masivo, con una masa crítica aportante suficiente, dota de valor al concepto de la financiación colaborativa y a la construcción de grandes colectivos en torno a proyectos concretos (Sajardo y Pérez, 2014).

Un ejemplo de la utilización de la tecnología blockchain para estas aplicaciones es la plataforma binance charity (www.binance.charity) ofrecida por la Binance Charity Foundation (BCF). En esta plataforma se presentan diferentes proyectos con impacto social positivo que han superado una due diligence realizada por el propio equipo técnico de la fundación, y que garantiza lo apropiado del proyecto para ser financiado en ella, buscando siempre la optimización del impacto social de cada unidad monetaria utilizada. La persona o entidad donante dispone de la posibilidad de elegir entre el conjunto de proyectos presentados, y a cuál de ellos quiere hacer llegar su donación. En el caso de que la donación no vaya dirigida a ningún proyecto específico, ésta pasa a gestionarse a través de la Binance Charity Wallet, la cual, de manera unilateral, realiza el reparto de los fondos recibidos.

Los costos operativos involucrados en las donaciones que se reciben a través de esta plataforma son asumidos por Binance (www.binance.com), una propuesta de ecosistema financiero en torno a la tecnología blockchain, de este modo se maximiza el porcentaje de donaciones que llegan a los beneficiarios finales.

Las donaciones pueden ser realizadas mediante la utilización de diferentes criptomonedas, lo cual permite la implementación de mecanismos de seguimiento de las donaciones por parte de cualquier persona, al ser el blockchain de Binance un blockchain público.

$\mathrm{BCF}$ es únicamente una muestra del potencial que el blockchain aporta al ámbito de las donaciones. Otros ejemplos que pueden ser tenidos en cuenta en este contexto son BitGive ${ }^{11}$, Bithope ${ }^{12}$, Helderbit ${ }^{13}$ o Givetrack $^{14}$. Igualmente existen propuestas de creación de monedas digitales con fines sociales para el apoyo a programas específicos, como por ejemplo Clean Water Coin $^{15}$, PinkCoin ${ }^{16}$ o AidCoin ${ }^{17}$.

Como puede observarse, la utilización de la tecnología blockchain en el ámbito de las donaciones cuenta con diversas propuestas que han adaptado la realidad de las plataformas de crowdfunding existentes para aprovechar todas las ventajas que esta tecnología aporta. La evolución de la propia tecnología y sus aplicaciones a buen seguro ejercerán de palanca para la construcción de nuevas propuestas en el ámbito de la financiación colectiva, ya sea desde la perspectiva de las donaciones o desde otras miradas, que en la actualidad aún se hallan en la imaginación, pero que indudablemente generarán continuas disrupciones en campos vinculados muy diversos.

\subsection{Inclusión financiera}

En 2018 el número de personas refugiadas que huían de conflictos bélicos en sus países de origen, incluso debido a situaciones de cambio climático que hacía enormemente difícil acceder a una vida digna, alcanzó la cifra de 70,8 millones de desplazados forzosos, el doble que hace 20 años (ACNUR, 2018a). La dimensión de esta realidad la ha situado "en el foco de atención de la sociedad civil global constituyendo un problema de urgencia politica y humanitaria" (Loewe, 2019:2).

Sin entrar a detallar y valorar las causas que provocan estos desplazamientos por no constituir este el objetivo del presente trabajo, sí se hace necesario poner en evidencia el conjunto de problemas a los que

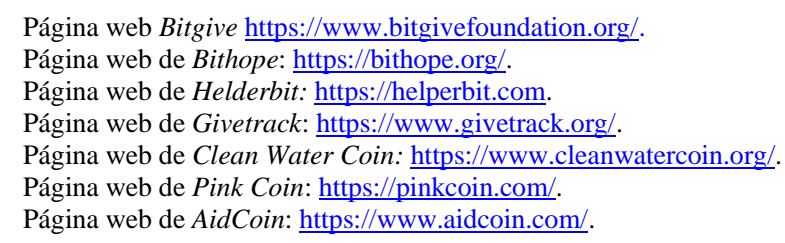


estos colectivos se enfrentan una vez arribados a un destino. Problemas determinantes para reconstruir sus vidas, ya sea de manera temporal o permanentemente, y que dificultan la normalización de una vida digna en los lugares de asentamiento.

Entre estos problemas se encuentra la incapacidad de acceder a servicios bancarios y financieros, bien por la falta de una prueba de identidad y/o arraigo en el lugar de destino, bien por las propias políticas de los países de acogida. Según la iniciativa Identification for Development (ID4D) del Banco Mundial (https://id4d.worldbank.org/) alrededor de mil millones de personas en todo el planeta carecen de una prueba de identificación oficial, que dificulta su acceso a múltiples servicios, entre ellos los bancarios. Esta situación merma la potencial integración de las personas refugiadas, su acceso al trabajo y a las finanzas, e indudablemente el que puedan apostar por el emprendimiento como salida económica (ACNUR, 2018b).

Ante esta situación, la asociación Arcadia Blockchain for Refugees ha construido la plataforma Arcadia a través de la cual se ofrecen recursos a organizaciones no gubernamentales (ONG) que trabajen el campo de las personas refugiadas, para proveer de servicios de transferencias de efectivo a este colectivo que vive una situación de exclusión financiera. Este colectivo es cuantitativamente muy significativo, tal y como evidencia un reciente estudio realizado por Arcadia según el cual, y como ejemplo, el $80 \%$ de los refugiados venezolanos no tenía acceso a servicios financieros. Esta exclusión surge principalmente por la negativa de las entidades financieras a la apertura de cuentas bancarias. La tecnología blockchain ofrece a estas personas la posibilidad de contar con una cuenta donde recibir y administrar su dinero, ello bajo la supervisión de la ONG que ofrece estos servicios a través de la plataforma Arcadia.

Según la propia página web de la comunidad Ethereum (https://ethereum.org/), esta es "la comunidad blockchain más grande y activa del mundo. Incluye desarrolladores de protocolos centrales, investigadores criptoeconómicos, cypherpunks, organizaciones mineras, titulares de ETH, desarrolladores de aplicaciones, usuarios comunes, anarquistas, compañias de Fortune 500...". La dimensión de esta comunidad permite garantizar que, en el caso de que la ONG a través de la cual está gestionando su cuenta con Arcadia dejase de ofrecer el servicio por cualquier motivo, éste seguirá estando a disposición de las personas usuarias sin necesidad de intervención de la primera.

Este blockchain cuenta con una criptomoneda, el Ether (ETH), que es el elemento que va a ser utilizado para transferir, a las personas usuarias de este sistema, el valor con el que realizar pagos. Igualmente, la elección de Ethereum garantiza la existencia de una gran comunidad entre personas desarrolladoras y usuarias que constituye un ecosistema mainstream que viene funcionando desde el año 2015.

El objetivo de esta entidad es posibilitar la inclusión de estos colectivos en la economía local del lugar donde fijan su residencia, ya sea temporal o definitiva, en condiciones de igualdad. Para ello, Arcadia ha creado herramientas de fácil comprensión y uso, incluso para personas no familiarizadas con las criptomonedas y su operativa, con el fin de dotar de sencillez a todo el proceso de recepción de fondos por parte de las personas refugiadas, que constituyen por el momento su target principal de usuarios, y su utilización en sus transacciones económicas reales.

Esta operativa comienza con el registro de la persona que va a utilizar esta forma de pagos en la plataforma Arcadia. Para ello únicamente es necesario una cuenta de correo electrónico, y una dirección postal a la que la entidad gestora de las aplicaciones de Arcadia remitirá una tarjeta física en la que se incluye la clave pública de la billetera de ETH que se emite en este mismo acto, y en otra tarjeta complementaria la clave privada de esa misma cuenta. La citada clave pública es válida para acceder a la plataforma y realizar toda la operativa necesaria, como revisar el balance de la billetera a través de la cual la persona usuaria recibe los fondos, obtener esos fondos e incluso compartirlos con otras personas que puedan precisarlos. La clave privada es necesaria para poder realizar transacciones y pagar por productos y servicios, siendo utilizada conjuntamente con una frase de contraseña que la protege de un uso inadecuado en caso de pérdida.

Igualmente, y para el acercamiento del cobro de productos y servicios se utiliza el ETH como unidad de valor. Para ello, Arcadia provee la plataforma www.arcadiapay.net que hace comprensible a los comerciantes, sin conocimientos técnicos sobre blockchain, la utilización del ETH como medio de pago. A tal efecto, simplemente deben registrar su negocio en esta aplicación, asociando en él una billetera de ETH en la que recibir los pagos, e indicar la moneda fiat, que puede ser en euros, dólares u otras divisas, en la que están marcados los precios que van a ser utilizados en las transacciones.

Este esquema de funcionamiento, y como es de esperar al estar basado en una tecnología que se encuentra todavía en una fase de desarrollo incipiente, contiene determinados inconvenientes que deben ser tomados en consideración a la hora de ser utilizados.

Las criptomonedas en general, salvo aquellas conocidas como stablecoins que han sido diseñadas para mantener un valor estable frente a divisas fiat, como es el DAI (aquí quito las referencias al as webs de estas cripto) o el USDT, presentan un determinado componente de volatilidad que introduce un cierto factor de incertidumbre en este esquema. Para minimizar estos efectos Arcadia propone a los diferentes usuarios realizar conversiones de ETH a la moneda fiat en un tiempo razonablemente corto y obtener así un cambio cercano a aquel existente en el momento de realizar la transacción. En el futuro plan de acción de Arcadia se 
contempla la obtención de la licencia necesaria para realizar directamente los cambios de criptomoneda a divisas fiat, para posteriormente lanzar un token propio, diseñado con la visión de stablecoin, referenciado a diversas monedas fiat. Todo ello es posible gracias a las posibilidades que el blockchain de Ethereum ofrece para la creación de tokens propios en un formato estándar (ERC20), que los habilita para ser utilizados en la construcción de nuevas aplicaciones utilizando para ello contratos inteligentes.

\subsection{Renta básica universal}

La Renta Básica Universal (RBU) consiste en la transferencia periódica e incondicional de renta a todas las personas de una sociedad que se hallen en condiciones de pobreza y exclusión, y ello en términos de un derecho establecido (Casassas y Raventós, 2011). Queda fuera de este trabajo el análisis de su pertinencia o la posibilidad de su implementación en este siglo XXI, existiendo un más que nutrido número de trabajos a este respecto al que se remite al lector. No obstante, el concepto de RBU ha sido considerado en este estudio puesto que la tecnología blockchain habilita un corpus de herramientas que van a posibilitar, conjuntamente con la voluntad de los miembros de una sociedad, construir ecosistemas sociales y económicos alternativos donde se contemple el concepto de la RBU.

En el núcleo mismo de estos nuevos ecosistemas existe la posibilidad de introducir dinámicas de funcionamiento alternativas que den un sentido diferente al concepto de valor de los bienes tangibles y al de las relaciones humanas. Éstas dinámicas se basan en el establecimiento de sistemas de creación y distribución de criptomonedas automáticos y descentralizados que garanticen estadios de igualdad entre las diferentes personas participantes.

Bien es cierto que tales propuestas pueden observarse como un intento de cuestionar el statu quo actual, no obstante y con independencia de ello, lo relevante es que en su seno se hallan diferentes propuestas que nacen al abrigo de criptomonedas creadas ad hoc, así como de un conjunto de contratos inteligentes en base a los cuales se definen las reglas de distribución y utilización de las mismas (Pérez y Sajardo, 2018).

En el espacio de la tecnología blockchain existen diferentes propuestas que giran en torno al concepto de la RBU y a su implementación que utilizan esta tecnología como vía de solución a la desigualdad de ingresos de las personas y como herramienta de lucha contra la pobreza extrema.

Un ejemplo, que dispone de una relevante trayectoria, lo constituye el proyecto $U B U$ (www.projectubu.com). Se trata de una iniciativa que ha desarrollado una DAO (organización autónoma descentralizada) y que distribuye diariamente 100 tokens UBU, a través de la una billetera digital específica para este valor, entre todas las personas que se hayan registrado como citizen (ciudadano) en la aplicación creada a tal efecto. Paralelamente, el proyecto UBU construye una red de vendors (comerciantes) que utilizan esta plataforma como herramienta de promoción de sus marcas y productos, estableciendo diferentes estrategias de precios como por ejemplo la introducción de parte de los mismos en UBUs. Según se recoge en su página web "El proyecto UBU es un esfuerzo ambicioso para monetizar el despilfarro y las ineficiencias presentes en todos los ecosistemas económicos, y re-distribuir ese valor a todos los participantes en el mundo UBU".

Un UBU es un token gratuito que se distribuye diariamente entre todos los ciudadanos registrados en la plataforma, conformando la parte central de un ecosistema autónomo y descentralizado de RBU. El citado token puede ser utilizado para realizar transferencias de valor entre los ciudadanos y efectuar pagos en los comercios adheridos como opción alternativa a los medios tradicionales (cash, tarjetas de crédito y débito). Una de las principales características de esta propuesta es el proceso mediante el cual el balance de la billetera de cada ciudadano se ve reducido diariamente en un $1 \%$ de su volumen, el cual regresa al sistema para ser distribuido de nuevo entre los ciudadanos siguiendo el esquema habitual. Se puede entender este hecho como parte de un proceso de oxidación que incentiva la utilización de este token como instrumento de medida y de transferencia de valor que penaliza su acumulación. Este proceso surge desde el concepto creado Silvio Gesell (1862 - 1930) que se refiere a la caducidad o pérdida de valor del dinero con el paso del tiempo para así evitar su acumulación especulativa.

El proyecto $U B U$ en sus primeros estadios fue presentado como una herramienta de lucha contra la pobreza extrema en base a la creación de un nuevo ecosistema de ciudadanos y proveedores. El mismo se dotó de un conjunto de actores con herramientas que posibilitan extraer valor a los activos moribundos (productos no aceptados por el mercado tradicional bien por la cercanía de su caducidad, su diseño fuera de tendencias actuales, ser artículos ya utilizados, etc), e inyectarlos nuevamente en el propio ecosistema creando una red de distribución equitativa de riqueza (Pérez y Sajardo, 2018). En la actualidad los fundamentos de funcionamiento del proyecto mantienen la distribución automática y descentralizada de los UBUs con un objetivo centrado en el establecimiento de relaciones con marcas comerciales que introduzcan su utilización en sus estrategias de marketing.

Otro ejemplo de utilización de la tecnología blockchain en este campo se presenta bajo la denominación mannabase. Esta propuesta lleva a cabo la distribución de una RBU entre todas las personas registradas en su plataforma y cuya identidad ha sido verificada. Para ello se utiliza una criptomoneda específica creada para 
tal efecto, el manna. Esta criptomoneda es la base de distribución de valor que se propone desde la Fundación People's Currency (https://www.prod.facebook.com/peoplescurrency/) como parte de un conjunto de herramientas construidas utilizando la tecnología blockchain, que bajo el denominador común de Hedge for Humanity (www.hedgeforhumanity.org) cubre los espacios de generación (humanity fund) y distribución (manna) de la riqueza, así como la identificación de las personas receptoras de las transferencias (www.brightid.org) como seres humanos únicos.

De igual forma a lo ocurrido con la propuesta del proyecto UBU, se ha llevado a cabo una reflexión estratégica y de sostenibilidad en torno al conjunto del proyecto en sí. Reflexiones que han tenido mucho que ver con la escalabilidad del proyecto y su sostenibilidad en base a los costes que la utilización de la propia tecnología implica. En este último sentido deben ser identificados el coste inherente al mantenimiento de los servidores que alojen las hot wallets que son utilizadas para la distribución de activos, y la falta de lucratividad para los mineros de bloques de mannabase en un blockchain exclusivo, que obliga a la migración a otra cadena de bloques más robusta y escalable (aqui quito la nota al pie). El proyecto sigue siendo un proyecto experimental que se ha financiado con los fondos aportados por sus fundadores, y merced de la dedicación de voluntarios y simpatizantes. Como perspectiva futura este grupo de actores se centra en la búsqueda de una mayor financiación que permita un escalado del proyecto a un nivel tal que pueda tener un impacto significativo en la vida de las personas.

Por último, un tercer ejemplo que merece consideración dentro de esta misma línea es The GoodDollar. Esta propuesta se presenta como un programa de investigación centrado en la reducción de la desigualdad en base a la utilización adecuada de la tecnología blockchain. Para ello, gracias a la participación de personas voluntarias como usuarias del servicio, se realiza un test de distribución de su criptomoneda. Según se detalla en su página web, su misión es “...construir una nueva criptomoneda global de código abierto, denominada GoodDollar, que sirva para distribuir dinero mediante la utilización de los principios de la RBU.

Se trata de un proyecto sin ánimo de lucro que, al igual que los proyectos considerados con anterioridad en este apartado, tiene como objetivo fomentar la creación de un ecosistema alternativo dirigido a reducir la desigualdad entre las personas desde una perspectiva económica.

Las áreas de investigación del proyecto GoodDollar se dirigen a la búsqueda de soluciones a los desafíos que condicionan el funcionamiento de una RBU a escala global. Estas áreas son:

1. Identidad social, garantizando la identidad única de la persona usuaria en el paradigma de la tecnología blockchain.

2. Interés social, implementando un sistema en el que la participación y el compromiso de la comunidad sea capaz de crear una plusvalía.

3. Gobernanza Social, buscando un modelo de gobernanza que permita a las comunidades, independientemente de su tamaño, la autogestión de su economía.

Todas y cada una de las iniciativas consideradas en este apartado, conforman proyectos generados inicialmente con el claro de luchar directamente contra la desigualdad y la pobreza mediante la implementación de una RBU, y que en la actualidad trabajan en una línea con un objetivo común, como es alcanzar una redistribución de la riqueza en base a sistemas tecnológicos automáticos, descentralizados y universales. Con ello, y adoptando los principios tradicionales de la RBU para aplicarlos a sus propias criptomonedas, proporcionar a las personas más necesitadas un recurso de apoyo financiero sólido.

\section{Conclusiones}

El conjunto de problemas globales a los que se enfrenta la sociedad del siglo XXI requiere un abordaje complejo, que analice sus orígenes y haga frente a sus efectos desde una perspectiva sistémica y sostenible, y donde se hace imprescindible la aplicación de instrumentos basados en la innovación social.

La centralización en la gobernanza de muchas esferas de nuestra vida, la asimetría informativa o la falta de confianza son ejemplos de factores que han venido provocado consecuencias muy significativas en nuestras sociedades, como son el incremento de la desigualdad, la falta de respeto de los derechos humanos, o las agresiones al medio ambiente con el consecuente cambio climático al que hoy de manera evidente nos enfrentamos.

La mencionada centralización ha venido dirigida por dos factores principales, a saber, la carencia de tecnología que permitiera con garantías una descentralización procesos de diversa índole, y la lucha por el mantenimiento del statu quo de los poderes ya establecidos.

$\mathrm{Y}$ este contexto se simultánea con un proceso constante de innovación tecnológica que ha permitido ofrecer herramientas digitales sin precedentes. Una de estas herramientas es el blockchain, una tecnología construida mediante a la unión de tecnologías dirigidas a garantizar la integridad de la información, transmitirla entre pares de un modo directo, estructurar los datos en forma de árbol, dotando con ello de eficiencia la organización de la información y facilitar su comprobación y acceso, el uso de pruebas de 
consenso y también de contratos inteligentes. Todas ellas son piezas de un amplio puzzle que conforma el blockchain, una tecnología que permite establecer bases de datos descentralizadas, transparentes, de solo escritura, e inalterables en el tiempo.

A lo largo del periodo de expansión de las plataformas y aplicaciones que utilizan esta tecnología, cabe resaltar que ésta se ha venido desarrollando en términos de código abierto, base de su visión descentralizada, transparente y accesible, todo lo cual ha impulsado un más que notable y amplio desarrollo.

El inicio del despliegue de esta tecnología se ha realizado principalmente en el campo de las finanzas a través de la creación de activos digitales a los que se les ha asociado un valor financiero y todo un conjunto de servicios en torno a él. Este nuevo escenario se ha visto impulsado por la crisis financiera iniciada en septiembre de 2008 que supuso el origen de una crisis de confianza sin precedentes en las instituciones, incluyendo entre ellas la banca, y que ha venido a constatar cómo la sociedad, las personas, son capaces de asignar valor a un activo sin ningún tipo de respaldo más que la propia confianza en el mismo.

No obstante, la realidad nos muestra como el potencial de la tecnología blockchain va mucho más allá de la mera actualización o modernización de los sistemas financieros, ya que con la aparición de los contratos inteligentes por ella utilizados, la diversidad de la oferta de servicios construidos ha crecido de un modo continuado. Una oferta que incluye desde los servicios más tradicionales que han utilizado la tecnología blockchain simplemente para introducir ciertas mejoras de gobernanza y confianza en las aplicaciones de tracking que vienen siendo utilizadas por operadores de la distribución de diferentes sectores, pero también otros completamente nuevos y cuyo desarrollo solo adquiere sentido gracias a esta tecnología. Este es el caso de los ecosistemas que surgen aunando en un mismo entorno un conjunto de aplicaciones distribuidas que ofrecen servicios diversos, un token digital intercambiable y estrategias de red basadas en las redes sociales tradicionales. Dos ejemplos claros de este tipo de propuestas son pillar project (https://pillarproject.io/) o coinbase wallet (https://wallet.coinbase.com/).

Estas aplicaciones descentralizadas abren la puerta a la aparición de herramientas de gestión del comportamiento humano desde la perspectiva de la puesta en valor de sus acciones de impacto social positivo. Ejemplos de las mismas han sido puestos en evidencia y analizado a lo largo de este trabajo en los ámbitos de la democratización de la producción energética, de la inclusión financiera, de la financiación colectiva o de la RBU, ejemplos que también pueden hallarse en ámbitos muy diversos como el reciclaje de plásticos, de materia orgánica, o para la realización de ejercicio físico. La monetización, tanto para la entidad desarrolladora como para las personas usuarias de estas aplicaciones, se basa en diferentes estrategias de mercado que escapan al ámbito del presente trabajo.

La tecnología blockchain se encuentra todavía en una fase muy incipiente de desarrollo y precisa de mejoras en las áreas de estandarización de los desarrollos, seguridad y regulación. Y es que existe una disparidad importante entre las diferentes cadenas de bloques existentes en el ciberespacio, por lo que su estandarización facilitará la interacción entre diferentes blockchains aportando eficacia tanto a estas interacciones como al desarrollo de las aplicaciones creadas en la misma cadena de bloques. Del mismo modo, la falta de un marco legal en torno a ella crea una situación de inseguridad jurídica, similar a la acontecida en las primeras fases de desarrollo de las plataformas de financiación participativa o crowdfunding, que resulta condicionante para su desarrollo. La generación de todo este marco normativo va a ser decisiva para un despliegue más ágil de esta tecnología en una atmósfera de seguridad, generadora de confianza para los diferentes grupos de interés que surjan en torno a cada una de las propuestas que vayan apareciendo.

Vislumbrar el futuro de la tecnología blockchain se torna complicado más allá del convencimiento evidenciado, a lo largo de este trabajo, de su implantación en muchos de los procesos de intercambio tanto de valor mediante los diferentes tokens fungibles ${ }^{18}$ que existen en la actualidad y la continua aparición de nuevos tokens a lo largo del tiempo, como de activos físicos mediante la utilización de los tokens no fungibles ${ }^{19}$. Sin embargo, el número de aplicaciones de esta tecnología que aparecen fuera del ámbito meramente financiero son cada vez más numerosas y aportan, gracias a sus características, soluciones distribuidas, transparentes y seguras a una gran variedad de problemáticas económicas, sociales y ambientales.

La visión que subyace tras esta tecnología apuesta por una perspectiva de valores que va más allá de la concentración del poder económico que se produce en el sistema capitalista dominante, abriendo la puerta a la construcción de un nuevo paradigma en el que las referencias al valor de las cosas, de los comportamientos y del mismo intercambio se construye con bases diferentes a las tradicionales, y con instrumentos de transacción que no tienen por qué tener una referencia directa con los existentes en la actualidad.

18 Un token es fungible si elementos o cantidades de dicho tipo o similar tipo pueden ser completamente diferenciables, por ejemplo las criptomonedas.

19 Tokens no fungibles son aquellos que presentan características únicas que hacen posible su distinción de otros tokens del mismo tipo y su trazabilidad, por ejemplo un token que represente la propiedad de una vivienda. 
Si bien habitualmente las ventajas de las nuevas tecnologías suelen favorecer a las personas consumidoras que cuentan con una situación personal de patrimonio y conexión, la tecnología blockchain puede dirigirse hacia la creación de oportunidades para la parte de la ciudadanía más débil y/o marginada. En este sentido van a jugar un papel relevante los elementos que forman parte del marco de trabajo de la comunidad criptográfica creadora de estas nuevas oportunidades. Así, existen cada vez más iniciativas de utilización de esta tecnología en ámbitos de impacto social auspiciados por actores relevantes en esta esfera, como es el caso de UNICEF. Del mismo modo estas aplicaciones de impacto pueden facilitar el camino para el alcance de los objetivos de desarrollo sostenible (ODS) de la agenda 2030, al poner en manos de la sociedad civil herramientas descentralizadas que le permitan atender las demandas de acción que esto conlleva.

La tecnología blockchain se encuentra en una fase inicial, pero de rápido desarrollo, y está siendo utilizada en campos muy diversos. La velocidad de crecimiento de esta herramienta transformadora va a depender de cómo se solucione el déficit de personas desarrolladoras y programadoras con suficiente conocimiento sobre ella existentes en el mercado, del marco regulatorio que se establezca en torno a la misma, y como no puede ser de otro modo, de la visión que las personas emprendedoras tengan frente a los problemas sociales y ambientales y su disposición a enfrentarse a ellos, puesto que blockchain es sólo la herramienta.

\section{Nota de los autores}

La velocidad de evolución del universo blockchain al igual que los difíciles momentos que está viviendo la sociedad mundial con motivo de la pandemia provocada por el virus COVID-19 hace que dos de los casos presentados en este artículo hayan sido clausurados. Nos referimos al Proyecto UBU y al Arcadia. Si bien las url que se referencían en ambos proyectos no contienen ya la información relativa a los mismos, los autores del presente artículo consideran adecuado el mantenimiento de los mismos en el texto del mismo por la validez, cada vez más, de los principios sobre los que se sustentaban estos proyectos y de las soluciones innovadoras que se crearon para atenderlos.

\section{Referencias bibliográfícas}

ACNUR (2018a) Informe anual. Agencia de la ONU para los refugiados. Disponible en: https://eacnur.org/files/reports/file/informe_anual_de_acnur_2018.pdf.

ACNUR (2018b) Medios de Vida e Inclusión Económica para Personas Refugiadas. Nota Conceptual: Estrategia Global 2019-2023. Disponible en: https://www.acnur.org/es-es/5c00562d4.pdf.

Acuña, H. (2018) Criptomonedas, aplicaciones potenciales de Blockchain y desafíos regulatorios. Universidad de los Andes, $\mathrm{N}^{\circ} 2$, pp. 1-51. Disponible en:

https://www.ese.cl/ese/site/artic/20181030/asocfile/20181030144442/cuaderno_2 cef acu a 2018 criptomone das aplicaciones potenciales de blockchain_y desaf os_regulatorios.pdf.

Antonopoulos, A. M. y Wood, G. (2018) Mastering ethereum: building smart contracts and dapps. Newton, Massachussets: O'Reilly Media. ISBN: 9781491971949.

Arango, J. (2003) La explicación teórica de las migraciones: luz y sombra. Migración y desarrollo. $\mathrm{N}^{\mathrm{o}} 1$. DOI:10.35533/myd.0101.ja.

Ayuso, M. y Holzmann, R. (2014) Natalidad, pirámide poblacional y movimientos migratorios en España: su efecto en el sistema de pensiones. Documentos de trabajo del Instituto BBVA de pensiones, $\mathrm{N}^{\mathrm{o}}$ 8, pp.1-18. Disponible en: https://www.researchgate.net/profile/Mercedes_Ayuso/publication/268215020_Natalidad_piramide_poblacional_y_ movimientos_migratorios_en_Espana/links/54747d730cf29afed60f8278.pdf.

Barricarte, J. J. S. (2011) Socioeconomía de las migraciones en un mundo globalizado. Reis, No 133, pp. 81-94. Disponible en: http://www.reis.cis.es/REIS/PDF/Reis 133 061295262072948.pdf.

Buterin, V. (2013) Ethereum White Paper: A Next-Generation Smart Contract and Decentralized Application Platform. Disponible en: https://github.com/ethereum/wiki/wiki/White-Paper.

Costa, M. T. (2016) Evolución del sector eléctrico español (1975-2015). Información Comercial Española. Revista de Economía ICE, No 889-890, pp.139-156.

Crosby, M., Pattanayak, P., Verma, S., y Kalyanaraman, V. (2016) Blockchain technology: Beyond bitcoin. Applied Innovation Review, $\mathrm{N}^{\circ} 2$, pp. 6-10.

Del Val Román, J.L. (2016) Industria 4.0: la transformación digital de la industria" Conferencia de Directores y Decanos de Ingeniería Informática. Facultad de Ingeniería de la Universidad de Deusto.

De Pedro, F.; Cid, J.Á. Cid y Casas, P. T. (2006) Las remesas de emigrantes en la Balanza de Pagos española. Boletín Económico. Disponible en:

https://www.bde.es/f/webbde/SES/Secciones/Publicaciones/InformesBoletinesRevistas/BoletinEconomico/06/JulAg o/Fich/art7.pdf.

Edelman E.T. (2019) Edelman Trust Barometer Global Report. Disponible en: 
https://www.edelman.com/sites/g/files/aatuss191/files/2019-03/2019_Edelman_Trust_Barometer_Global_Report.pdf ESADE, PwC y La Caixa, F. (2013): Estudio sobre el presente y futuro del Tercer Sector social en un entorno de crisis. https://www.pwc.es/es/fundacion/assets/presente-futuro-3sector.pdf.
INE (2018)
España
en
cifras
2018.
Disponible
en:

https://www.ine.es/prodyser/espa_cifras/2018/files/assets/common/downloads/publication.pdf.

Espinosa, J. y Campillo, I. (2018) La precarización en su contexto: desarrollo y crisis del régimen de empleo en España. Papeles de relaciones ecosociales y cambio global, $\mathrm{N}^{\circ} 140$, pp.51-63.

Fernández, M.; Franco, L., y Meixide, A. (2013) La reestructuración bancaria en Galicia: impacto sobre la exclusión financiera y territorial. XXXIX Reunión de Estudios Regionales, Oviedo, 21-22 de noviembre.

Frey, C. B. \& Osborne, M. A. (2017) The future of employment: how susceptible are jobs to computerisation? Technological forecasting and social change, $\mathrm{N}^{\circ} 114$, pp.254-280. DOI: 10.1016/jtechforce.2016.08.019.

García Ruiz, M. (2015) De la quiebra del Lehman Brothers a la crisis de la deuda soberana en Europa: el quinquenio gris de los mercados financieros internacionales. Economía y Desarrollo, № 154, Vol. 1, pp.45-59.

Grande, R. (2018) ¿Integración o remesas? Determinantes de las transferencias monetarias de los migrantes latinoamericanos en España. Revista Desarrollo y Sociedad, № 80, pp.155-187. DOI: 10.13043/DYS.80.5.

Hernández, R.; Fernández, C. \& Baptista, P. (2010) Metodología de la investigación. México: McGraw Hill, Mexico. ISBN: 978-1-4562-2396-0.

Iansiti, M. y Lakhani, K. R. (2017) The truth about blockchain. Harvard Business Review, No 95, Vol. 1, pp.118-127.

Kahale, C. y Djamil, T. (2017) La industria 4.0: los retos para el empleo español. En Mella, L. (2017) Los actuales cambios sociales y laborales: nuevos retos para el mundo del trabajo. I, Cambios tecnológicos y nuevos retos para el mundo del trabajo:(Portugal, España, Colombia, Italia, Francia). Berlin: Peter Lang.

Loewe, D. (2019) Refugiados, desplazados e inmigrantes económicos. El caso de la dignidad. Veritas (Porto Alegre), No 64, Vol. 1. DOI: 10.15448/1984-6746.2019.1.33464.

Martínez Carazo, C. P. (2006) El método de estudio de caso: estrategia metodológica de la investigación científica. Pensamiento \& Gestión, No 20, pp. 165-193. ISSN:1657-6276. Disponible en: https://www.redalyc.org/pdf/646/64602005.pdf.

Moré, I. (2005) Las remesas de los emigrantes en España: una oportunidad para la acción exterior”, Boletín El Cano, DT N³. ISSN: 1696-332. Disponible en: http://biblioteca.ribei.org/943/1/DT-003-2005.pdf.

Nakamoto, S. (2008) Bitcoin: A peer-to-peer electronic cash system. Disponible en: https://bitcoin.org/bitcoin.pdf.

Pérez Díaz, J. (2010) El envejecimiento de la población española. Investigación y Ciencia, N 410, pp.34-42.

Pylon Network (2018) The energy blockchain platform white paper. Disponible en: https://pylon-network.org/wpcontent/uploads/2019/02/WhitePaper_PYLON_v2_SPANISH-1.pdf.

Pérez, S. (2017) La nueva filantropía: hacia la era de la sociedad mecenas. Análisis del crowdfunding social en España. Doctoral dissertation. Universitat de València.

Pérez, S. \& Sajardo, A. (2018) Herramientas de economía colaborativa en la implementación de la RBU: el potencial del blockchain. Congreso Internacional sobre Economía Colaborativa, Economía Social y Bienestar, Valencia 29 y 30 de noviembre.

Ruiz, R. (2015) El Tercer Sector de Acción Social en 2015: impacto de la crisis. Plataforma de ONG de Acción Social y Plataforma del Tercer Sector, Madrid. Disponible en:

https://repositorio.comillas.edu/xmlui/bitstream/handle/11531/15078/estudio_completo_el_TSAS_en_2015_impact o de la crisis.pdf? sequence $=1 \&$ is Allowed $=y$.

Rohr, J. \& Wright, A. (2017) Blockchain-based token sales, initial coin offerings, and the democratization of public capital markets. Cardozo Legal Studies Research Paper, N 527.

Roth, F. (2009) The effect of the financial crisis on systemic trust. Intereconomics. No 44, Vol. 4, pp. $203-208$.

Sajardo, A. (2012) Análisis económico de Sector No Lucrativo. Aspectos económicos del voluntariado. Valencia: Tirant Lo Blanch.

Sajardo, A. (2016) Nuevos instrumentos de financiación para el Sector No Lucrativo. El reto del crowdfundig social. Revista Cooperativismo \& Desarrollo, № 24, Vol. 108, pp. 39-54. DOI: 10.16925/co.v24i108.1259.

Sajardo, A. \& Pérez, S. (2014) El sector No Lucrativo ante las nuevas herramientas de fundraising: ¿el crowdfunding antesala de la sociedad mecenas? Revista Vasca de Economía Social, No 13, pp.149-184.

Sajardo, A. \& Chaves Sajardo, R.J. (2015) Reingeniería socioeconómica al servicio del Sector No Lucrativo: el caso de crowdfundig social en España. XVIII Congreso AECA, Cartagena 30 septiembre a 2 octubre.

Sajardo, A. \& Pérez, S. (2015) Towards The movilization of social margins: Noprofit Sector and informtion comunication technology. 5th CIRIEC International Research Conference on Social Economy, Lisbon, July the 15th to 18 th.

Sajardo, A. \& Pérez, S. \& Gil, M. (2017) El Sector No Lucrativo en el marco de la nueva era tecnológica. El desafío del crowdfunding social en España. El caso de la asociación Alba Pérez, lucha contra el cáncer infantil. REVESCO. Revista de Estudios Cooperativos, Segundo Cuatrimestre, No 124, pp. 128-151. DOI: 10.5209/REVE.56132.

Sajardo, A. \& Pérez, S. (2019) Análisis de las plataformas de crowdfundig social en el estado español. REVESCO. Revista de Estudios Cooperativos, Primer Cuatrimestre, $\mathrm{N}^{\mathrm{o}}$ 130, pp. 149-175. DOI: http://dx.doi.org/10.5209/REVE.61938.

Schneiders, A., \& Shipworth, D. (2018) Energy Cooperatives: A Missing Piece of the Peer-to-Peer Energy Regulation Puzzle? Available at SSRN 3252486. 
Szabo, N. (1997) Formalizing and securing relationships on public networks. First Monday, № 2 , Vol. 9. DOI:10.5210/fm.v2i9.548.

Tsvetanova, B. M. (2017) Las remesas de los inmigrantes en España: el marco jurídico español e internacional. Tesis Doctoral. Universidad Complutense de Madrid.

Villarreal, O. \& Landeta, J. (2010) El estudio de casos como metodología de investigación científica en dirección y economía de la empresa. Una aplicación a la internacionalización. Investigaciones europeas de dirección y economía de la empresa, № 16, Vol. 3, pp. 31-52. DOI: 10.1016/S1135-2523(12)60033-1.

Wüst, K., \& Gervais, A. (2018) Do you need a Blockchain?, Crypto Valley Conference on Blockchain Technology (CVCBT), IEEE, pp. 45-54.

Yin, R. K. (1989) Research design issues in using the case study method to study management information systems. The information systems research challeng. Qualitative research methods. No 1, pp. 1-6.

Yli-Huumo, J.; Ko D.; Choi, S.; Park, S. \& Smolander, K. (2016) Where Is Current Research on Blockchain Technology? A Systematic Review. PLoS ONE, No 11 (10): e0163477. DOI: 10.1371/journal.pone.0163477. https://doi.org/10.1371/journal.pone.0163477.

Zhenqian, H. (2018) Universal Basic Income, MPDD Policy Briefs PB66, United Nations Economic and Social Commission for Asia and the Pacific (ESCAP). Disponible en: https://ideas.repec.org/p/unt/pbmpdd/pb66.html

Zubeldia, A. M.; Grajirena, J. M.; Sogorb, A. O. \& Zubiaurre, M. Z. (2008) Reflexiones sobre el origen y las implicaciones de la exclusión financiera. Universidad, sociedad y mercados globales. Asociación Española de Dirección y Economía de la Empresa (AEDEM), pp. 209-218. ISBN: 978-84-691-5667-4. 